\title{
Desarrollo de un controlador predictivo con compensación por adelanto y garantía de estabilidad: Resultados preliminares
}

\author{
Igor M. L. Pataro ${ }^{a}$, Juan D. Gil ${ }^{a}$, Marcus V. Americano da Costa $^{b}$, José L. Guzmán ${ }^{a}$, \\ Manuel Berenguel ${ }^{a}$ \\ ${ }^{a}$ Centro Mixto CIESOL, ceiA3, Universidad de Almería. Ctra. Sacramento s/n, Almería 04120, Spain \\ ${ }^{b}$ Departamento de Ingeniería Química, Universidad Federal de Bahia, R. Prof. Aristídes Novis, 2, \\ Salvador, Bahia, Brazil

\begin{abstract}
Correos: ilp428@inlumine.ual.es (Igor M. L. Pataro), juandiego.gil@ual.es (Juan D. Gil), marcus.americano@ufba.br (Marcus V. Americano da Costa), joseluis.guzman@ual.es (José L. Guzmán), beren@ual.es (Manuel Berenguel).
\end{abstract}

\section{Resumen}

Uno de los aspectos esenciales en el diseño de un sistema de control consiste en garantizar su estabilidad en bucle cerrado. Este artículo presenta un controlador predictivo basado en modelo de horizonte infinito con compensación por adelanto y garantía de estabilidad en bucle cerrado para sistemas estables. La estabilidad del controlador se garantiza mediante los conceptos de solución recursiva y disminución asintótica de la función de coste. La técnica desarrollada se prueba en simulación usando como sistema de referencia un campo de captadores solares de placa plana. Además, se presenta una comparación con el mismo enfoque de control pero sin incluir la compensación por adelanto. Los resultados obtenidos demuestran que la estrategia propuesta mejora el desempeño del controlador rechazando correctamente las perturbaciones y manteniendo las características de estabilidad nominal, lo que asegura la convergencia en bucle cerrado tanto para los problemas de seguimiento de referencia como de rechazo de perturbaciones.

Palabras clave: Control predictivo basado en modelo, control por adelanto, control predictivo con horizonte infinito, energía solar.

\section{INTRODUCCIÓN}

Las estrategias de control predictivo se basan en la minimización de un determinado criterio para mantener la salida del proceso lo más cerca posible a una trayectoria de referencia. Este criterio toma normalmente la forma de una función cuadrática del error entre la salida predicha y la trayectoria de referencia futura. Así, la función a minimizar depende esencialmente de un modelo del sistema, el cual se usa para predecir la salida de este a lo largo del horizonte de predicción considerado y de una serie de parámetros de ajuste, los cuales sirven para obtener el comportamiento deseado en bucle cerrado. Independientemente de estos parámetros, cuando el modelo del sistema refleja fielmente el comportamiento de la planta real (caso nominal), se puede garantizar la estabilidad del controlador en bucle cerrado. Este tipo de controladores predictivos se conocen como estrategias de Control Predictivo basado en Modelo (MPC, por sus siglas en inglés) con garantía de estabilidad.

En la literatura, se pueden encontrar diferentes formulaciones MPC que garantizan la estabilidad en bucle cerrado del sistema de control [7]. Entre los diferentes enfoques, destacan las estrategias basadas en el uso de una función objetivo con horizonte de predicción infinito, las cuales se conocen como estrategias MPC de horizonte infinito (IHMPC, por sus siglas en inglés) [6]. En esta familia de controladores se pueden diferenciar dos categorías principales, (i) controladores por modo dual y (ii) controladores con restricción terminal, las cuales se diferencian entre sí en la formulación de la función objetivo y del propio problema de optimización [11]. Sin embargo, los enfoques basados en modo dual presentan varias limitaciones prácticas tales como un coste computacional alto, un dominio de atracción limitado, y un rendimiento del optimizador comprometido. Por este motivo, González et al. [4] propusieron añadir restricciones terminales para resolver los problemas de optimización incluidos en las técnicas IHMPC siguiendo las ideas presentadas en el trabajo de Rawlings y Muske [9] para sistemas lineales estables con restricciones politópicas. Por otro lado, la principal contribución del trabajo de Rodrigues y Odloak [10] fue la extensión de la formulación IHMPC para abordar de forma unificada los problemas de regulación y seguimiento para sistemas con polos estables, lo cual no se contempló en la formulación original de Rawlings y Muske [9]. Además, esta extensión aumentaba el dominio de atracción mediante el uso de variables de holgura que suvizan la restricción terminal del IHMPC, la cual asegura la factibilidad del problema de optimiza- 
ción. De este modo, la formulación de Rodrigues y Odloak [10] se considera un hito en el desarrollo de controladores de horizonte infinito, destacando las formulaciones para sistemas con polos integradores inestables, únicos y múltiples.

La madurez actual de las técnicas de control IHMPC con garantía de estabilidad hace que estas estrategias tengan un atractivo especial para diferentes aplicaciones industriales, como el caso de campos solares térmicos $[1,2,5]$. Sin embargo, este tipo de plantas se caracterizan por estar constantemente afectadas por variables externas difícilmente predecibles, como la irradiacia solar. Consecuentemente, las condiciones meteorológicas afectan directamente al sistema de control en forma de perturbación, lo cual justifica la búsqueda de técnicas que incluyan controladores por adelanto (FF, por sus siglas en inglés) para el rechazo de dichas perturbaciones.

Motivado por las necesidades específicas de control previamente mencionadas en plantas solares térmicas, este trabajo presenta un controlador predictivo nominal con compensación de perturbaciones y garantía de estabilidad. El controlador propuesto es una extensión de los trabajos de Rodrigues y Odloak [10] en el que se asegura la estabilidad nominal del controlador en base a los principios de solución recursiva y disminución asintótica de la función de coste. La estrategia se prueba en simulación usando como referencia un modelo validado de la planta termosolar del Centro Mixto CIESOL de la Universidad de Almería (España) [8] también utilizado en otros campos solares de placa plana en la Plataforma Solar de Almería [3] y se compara con un controlador basado en la formulación convencional IHMPC. Los resultados preliminares evidencian el buen desempeño de la técnica propuesta y las mejoras que se consiguen en presencia de perturbaciones medibles respecto a la formulación convencional.

Este trabajo se divide de la siguiente manera: La sección 2 presenta el modelo en estado de espacios parametrizado, incluyendo las perturbaciones medibles. La sección 3 detalla la formulación del controlador IHMPC con control por adelanto, el cual ser referirá en adelante como IHMPC+FF (por sus siglas en inglés). Además, describe la prueba del teorema que garantiza la estabilidad nominal del controlador. La Sección 4 presenta los resultados en simulación, comparando el IHMPC+FF propuesto y el IHMPC sin compensaciones por adelanto. Finalmente, la Sección 5 resume las principales conclusiones del trabajo.

\section{MODELO EN ESPACIO DE ESTADOS}

Esta sección presenta el modelado en espacio de estados extendido que se propone para la formulación del controlador IHMPC con compensación por adelanto. En primer lugar, se define el modelo de espacio de estados propuesto por Rodrigues y Odloak [10], el cual surge de la parametrización de la respuesta del sistema ante entradas en escalón:

$$
\begin{gathered}
{\left[\begin{array}{l}
\mathbf{x}^{i}(k+1) \\
\mathbf{x}^{s}(k+1)
\end{array}\right]=\underbrace{\left[\begin{array}{cc}
\mathbf{I}_{n y} & \mathbf{0} \\
\mathbf{0} & \boldsymbol{F}
\end{array}\right]}_{\mathbf{A}}\left[\begin{array}{l}
\mathbf{x}^{i}(k) \\
\mathbf{x}^{s}(k)
\end{array}\right]+\underbrace{\left[\begin{array}{l}
\mathbf{B}^{i} \\
\mathbf{B}^{s}
\end{array}\right]}_{\mathbf{B}} \boldsymbol{\Delta} \mathbf{u}(k)} \\
\mathbf{y}(k)=\underbrace{\left[\begin{array}{ll}
\mathbf{I}_{n y} & \mathbf{\Psi}
\end{array}\right]}_{\mathbf{C}}\left[\begin{array}{l}
\mathbf{x}^{i}(k) \\
\mathbf{x}^{s}(k)
\end{array}\right] .
\end{gathered}
$$

En este modelo, $\mathbf{x}^{s}$ y $\mathbf{x}^{i}$ reflejan, respectivamente, los estados integradores artificiales, que surgen por la formulación incremental de las entradas del modelo, y los estados estables, que provienen de los polos estables del sistema real. Las matrices auxiliares $\boldsymbol{F}$ y $\boldsymbol{\Psi}$ se asocian con la respuesta libre del sistema y las salidas respectivamente. Las matrices de coeficientes $\boldsymbol{B}^{\boldsymbol{i}}$ y $\boldsymbol{B}^{\boldsymbol{s}}$ provienen de la expansión de la función de transferencia del sistema en forma de fracciones parciales, lo cual representa la influencia de los incrementos de la señal de control $\boldsymbol{\Delta} \boldsymbol{u}$. Además, se definen $n y$, ns y $n u$ como el número de salidas, estados estables y entradas respectivamente.

Con base al modelo descrito anteriormente, el objetivo principal en este trabajo consiste en extender esta formulación para incluir el efecto de perturbaciones medibles. En esta extensión, se considera que las perturbaciones medibles no son variables manipulables del sistema, de forma que se mantiene el concepto básico del modelo presentado con anterioridad. Por lo tanto, el modelo en espacio de estados para la formulación del $\mathrm{IHMPC}+\mathrm{FF}$ viene dado por la siguiente expresión:

$$
\begin{aligned}
{\left[\begin{array}{l}
\mathbf{x}^{i}(k+1) \\
\mathbf{x}^{s}(k+1)
\end{array}\right]=} & \underbrace{\left[\begin{array}{cc}
\mathbf{I}_{n y} & \mathbf{0} \\
\mathbf{0} & \boldsymbol{F}
\end{array}\right]}_{\mathbf{A}}\left[\begin{array}{l}
\mathbf{x}^{i}(k) \\
\mathbf{x}^{s}(k)
\end{array}\right]+\underbrace{\left[\begin{array}{l}
\mathbf{B}^{i} \\
\mathbf{B}^{s}
\end{array}\right]}_{\mathbf{B}} \mathbf{\Delta} \mathbf{u}(k) \\
& +\underbrace{\left[\begin{array}{c}
\mathbf{D}^{i} \\
\mathbf{D}^{s}
\end{array}\right]}_{\mathbf{D}} \boldsymbol{\Delta} \mathbf{d}(k),
\end{aligned}
$$

$$
\mathbf{y}(k)=\underbrace{\left[\begin{array}{ll}
\mathbf{I}_{n y} & \boldsymbol{\Psi}
\end{array}\right]}_{\mathbf{C}}\left[\begin{array}{l}
\mathbf{x}^{i}(k) \\
\mathbf{x}^{s}(k)
\end{array}\right] .
$$


En este caso, $\boldsymbol{D}^{\boldsymbol{i}}$ y $\boldsymbol{D}^{\boldsymbol{s}}$ provienen de la descomposición en fracciones parciales de la función de transferencia de las perturbaciones, y nd representa el número de perturbaciones que afectan al sistema, las cuales se engloban en el vector $\boldsymbol{\Delta} \mathbf{d}$. Se debe remarcar nuevamente que las ecuaciones (3) y (4) son una extensión de modelo presentado por Rodrigues y Odloak [10] incluyendo solo el término $\boldsymbol{\Delta} \mathbf{d}$, sin alterar la formulación básica del modelo.

\section{DESARROLLO DEL CONTROLADOR IHMPC CON COMPENSACIÓN POR ADELANTO}

Considerando el modelado descrito en las ecuaciones (3) y (4), y siguiendo la formulación IHMPC con variables de holgura propuesta en [10], la estrategia convencional IHMPC se puede formular como sigue:

Problema 1:

$$
\min _{\boldsymbol{\Delta} \mathbf{u}_{k}, \boldsymbol{\delta}_{y, k}} J_{k, 1}
$$

donde:

$$
\begin{aligned}
J_{k, 1} & =\sum_{j=1}^{\infty}\left\|\mathbf{y}(k+j \mid k)-\mathbf{y}_{s p}-\boldsymbol{\delta}_{y, k}\right\|_{\mathbf{Q}_{y}}^{2} \\
& +\sum_{j=0}^{m-1}\|\boldsymbol{\Delta} \mathbf{u}(k+j \mid k)\|_{\mathbf{R}}^{2}+\left\|\boldsymbol{\delta}_{y, k}\right\|_{\mathbf{S}_{y}}^{2}
\end{aligned}
$$

sujeto a:

$$
\begin{gathered}
U=\left\{\begin{array}{c}
\Delta \mathbf{u}_{\min } \leq \boldsymbol{\Delta} \mathbf{u}(k+j \mid k) \leq \boldsymbol{\Delta} \mathbf{u}_{\max } \\
\boldsymbol{\Delta} \mathbf{u}(k+j \mid k)=0, j>m, \\
\mathbf{u}_{\min } \leq \mathbf{u}(k+j \mid k) \leq \mathbf{u}_{\max }
\end{array}\right. \\
\mathbf{x}^{i}(k \mid k)+\boldsymbol{B}_{m}^{i} \boldsymbol{\Delta} \mathbf{u}_{k}-\mathbf{y}_{s p}-\boldsymbol{\delta}_{y, k}=0
\end{gathered}
$$

donde

$$
\begin{array}{r}
\Delta \mathbf{u}_{k}=\left[\boldsymbol{\Delta} \mathbf{u}(k \mid k)^{\top} \boldsymbol{\Delta} \mathbf{u}(k+1 \mid k)^{\top} \ldots\right. \\
\left.\cdots \boldsymbol{\Delta} \mathbf{u}(k+m-1 \mid k)^{\top}\right]^{\top}, \\
\Delta \mathbf{d}_{k}=\left[\boldsymbol{\Delta} \mathbf{d}(k \mid k)^{\top} \boldsymbol{\Delta} \mathbf{d}(k+1 \mid k)^{\top} \cdots\right. \\
\left.\cdots \boldsymbol{\Delta} \mathbf{d}(k+m-1 \mid k)^{\top}\right]^{\top},
\end{array}
$$

$\mathbf{y}_{s p}$ es el vector de referencias del sistema y $m$ es el horizonte de control. En esta formulación, las variables de holgura $\boldsymbol{\delta}_{y, k}$ se utilizan para suavizar la restricción terminal descrita en la ecuación (7), y, además, contribuyen a incrementar el dominio de atracción del problema y a hacer que este siempre sea factible. Las matrices $\mathbf{Q}_{y}, \mathbf{R}$, y $\mathbf{S}_{y}$ son matrices de pesos que se usan como parámetros de ajuste del controlador. Nótese que en la matriz $\mathbf{S}_{y}$ se deben usar pesos con valores altos para forzar que solo se usen las variables de holgura cuando sean estrictamente necesarias. El teorema y la prueba de estabilidad del controlador formulado se puede consultar en el trabajo de Rodrigues y Odloak [10].

De este modo, y siguiendo la misma idea que el IHMPC, el problema de optimización considerado en el controlador IHMPC $+\mathrm{FF}$ viene dado por la siguientes ecuaciones:

\section{Problema 2:}

$$
\min _{\boldsymbol{\Delta} \mathbf{u}_{k}, \boldsymbol{\delta}_{y, k}} J_{k, 2}
$$

donde:

$$
J_{k, 2}=J_{k, 1}
$$

sujeto a:

$$
\text { Ec. (6), }
$$

$$
\mathbf{x}^{i}(k \mid k)+\boldsymbol{B}_{m}^{i} \boldsymbol{\Delta} \mathbf{u}_{k}+\boldsymbol{D}_{m}^{i} \boldsymbol{\Delta} \mathbf{d}_{k}-\mathbf{y}_{s p}-\boldsymbol{\delta}_{y, k}=0 .
$$

En esta formulación, la función objetivo es la misma que la que se usa en la formulación convencional del IHMPC. Sin embargo, los modelos usados en $J_{k, 2}$ son los presentados en las ecuaciones (3) y (4). Esta diferencia en los modelos hace que la restricción terminal de la formulación clásica IHMPC se debe modificar, añadiendo un nuevo término que considere el efecto de las perturbaciones tal y como se presenta en la ecuación (10).

La garantía de estabilidad del controlador IHMPC + FF viene dada por la prueba del siguiente teorema:

Teorema: Dado un sistema con polos estables y perturbaciones medibles, y considerando que las perturbaciones se pueden predecir a lo largo del horizonte de predicción; si en el instante de muestreo $k$ existe una solución factible del Problema 2, y el sistema se controla en el punto deseado en estado estacionario, entonces la solución al Problema 2 originará señales de control que llevarán a las salidas del sistema al punto de equilibrio deseado en bucle cerrado, siendo asintóticamente convergente.

Prueba: La prueba del Teorema sigue los mismos principios que los usados en el trabajo de Rawlings y Muske [9] y en el trabajo de Rodrigues y Odloak [10], los cuales se basan en la solución recursiva y convergencia asintótica de la función 
de coste. En primer lugar, para la solución recursiva, considerando que en el instante $k$ se obtiene la siguiente solución óptima:

$$
\begin{gathered}
\Delta \mathbf{u}_{k}^{*}=\left[\boldsymbol{\Delta} \mathbf{u}^{*}(k \mid k)^{\top} \boldsymbol{\Delta} \mathbf{u}^{*}(k+1 \mid k)^{\top} \ldots\right. \\
\left.\cdots \boldsymbol{\Delta} \mathbf{u}^{*}(k+m-1 \mid k)^{\top}\right]^{\top} \\
\boldsymbol{\delta}_{y, k}^{*},
\end{gathered}
$$

la cual produce un valor mínimo de la función de coste $J_{k}^{*}$, y considerando también que la señal de control $\boldsymbol{\Delta} \mathbf{u}^{*}(k \mid k)$ se implementa en la planta real usando una estrategia de horizonte deslizante, de modo que en el instante $k+1$ el Problema 2 se resuelve de nuevo. Al trabajar con un escenario nominal, la solución en el instante $k+1$ será una solución heredada de la solución en el instante $k$ :

$$
\begin{gathered}
\Delta \widetilde{\mathbf{u}}_{k+1}=\left[\boldsymbol{\Delta} \mathbf{u}^{*}(k+1 \mid k)^{\top} \boldsymbol{\Delta} \mathbf{u}^{*}(k+2 \mid k)^{\top} \ldots\right. \\
\left.\cdots \mathbf{0}^{\top}\right]^{\top}, \\
\widetilde{\boldsymbol{\delta}}_{y, k+1}=\boldsymbol{\delta}_{y, k}^{*} .
\end{gathered}
$$

Así, es fácil comprobar que $\widetilde{\mathbf{u}}_{k+1}$ puede satisfacer las restricciones del problema de optimización, ya que la única diferencia con el término $\Delta \mathbf{u}_{k}^{*}$ es el $\mathbf{0}$, el cual se incluye en todo el conjunto de restricciones. De modo que, para satisfacer la ecuación (10) considerando la restricción terminal en el instante $k+1$ :

$$
\begin{aligned}
& \underbrace{\mathbf{x}^{i}(k+1 \mid k+1)}_{\mathbf{x}^{i}(k+1 \mid k)}+\boldsymbol{B}_{m}^{i} \boldsymbol{\Delta} \widetilde{\mathbf{u}}_{k+1}+\boldsymbol{D}_{m}^{i} \boldsymbol{\Delta} \mathbf{d}_{k+1}-\mathbf{y}_{s p}-\widetilde{\boldsymbol{\delta}}_{y, k+1}=0 \\
& \underbrace{\mathbf{x}^{i}(k \mid k)+\boldsymbol{B}^{i} \boldsymbol{\Delta} \mathbf{u}^{*}(k \mid k)+\boldsymbol{D}^{i} \boldsymbol{\Delta} \mathbf{d}(k \mid k)}_{\mathbf{x}^{i}(k+1 \mid k)}+\boldsymbol{B}_{m}^{i} \Delta \widetilde{\mathbf{u}}_{k+1}+\boldsymbol{D}_{m}^{i} \boldsymbol{\Delta} \mathbf{d}_{k+1}-\mathbf{y}_{s p}-\widetilde{\boldsymbol{\delta}}_{y, k+1}=0 \\
& \underbrace{\mathbf{x}^{i}(k \mid k)+\boldsymbol{B}_{m}^{i} \Delta \mathbf{u}_{k}^{*}+\boldsymbol{D}_{m}^{i} \boldsymbol{\Delta} \mathbf{d}_{k}-\mathbf{y}_{s p}-\widetilde{\boldsymbol{\delta}}_{y, k}^{*}}_{E c .(7)=0}+\underbrace{\boldsymbol{D}^{i} \boldsymbol{\Delta} \mathbf{d}(k+m / k+1)}_{=\boldsymbol{\Delta} \mathbf{d}(k+m / k)=0}=0
\end{aligned}
$$

donde las matrices $\boldsymbol{D}_{m}^{i}$ y $\boldsymbol{B}_{m}^{i}$ se definen como sigue:

$$
\begin{aligned}
& \boldsymbol{B}_{m}^{i}=[\underbrace{\boldsymbol{B}^{i} \cdots \boldsymbol{B}^{i}}_{m}] \\
& \boldsymbol{D}_{m}^{i}=[\underbrace{\boldsymbol{D}^{i} \cdots \boldsymbol{D}^{i}}_{m}]
\end{aligned}
$$

Observación 1: De la ecuación (11), se puede deducir que los incrementos predichos de las perturbaciones deben ser cero al final del horizonte de control. Esta afirmación se puede satisfacer siempre y cuando se escojan valores bajos para el horizonte de control $m$, de forma que las variaciones en las perturbaciones sean muy pequeñas y se puedan despreciar.

Como en la solución heredada $\widetilde{\mathbf{u}}_{k+1}$ y $\widetilde{\boldsymbol{\delta}}_{y, k+1}$ son una solución factible, donde $\widetilde{J}_{k+1,2}$ es su coste asociado en el instante $k+1$. Se puede comprobar cómo la única diferencia en el valor de la función de coste en el instante $k+1$ y en instante $k$ es el primer término del vector de señales de control $\boldsymbol{\Delta} \boldsymbol{u}^{*}(k \mid k)$, que será el implementado en la planta real. Por tanto, las diferencias en la función de coste se pueden describir como sigue:

$$
\begin{aligned}
J_{k, 2}^{*}-\widetilde{J}_{k+1,2}= & \left\|\mathbf{y}(k+1 \mid k)-\mathbf{y}_{s p}(k+1)-\boldsymbol{\delta}_{y, k}^{*}\right\|_{\mathbf{Q}_{y}}^{2} \\
& +\| \boldsymbol{\Delta} \boldsymbol{u}(k \mid k))\left\|_{\mathbf{R}}^{2}+\right\| \boldsymbol{\delta}_{y, k}^{*} \|_{\mathbf{S}_{y}}^{2}
\end{aligned}
$$

Como $\boldsymbol{Q}_{\boldsymbol{y}}, \boldsymbol{R}$ y $\boldsymbol{S}$ son matrices definidas positivas, se puede comprobar como $J_{k, 2}^{*} \geq \widetilde{J}_{k+1,2}$. Consecuentemente, $\boldsymbol{\Delta} \widetilde{\mathbf{u}}_{k+1}$ y $\widetilde{\boldsymbol{\delta}}_{y, k+1}$ representan una solución factible para el Problema 2 en el instante $k+1$, de forma que $J_{k, 2}^{*} \geq \widetilde{J}_{k+1,2} \geq J_{k+1,2}^{*}$. Por tanto, la función de coste $J_{k, 2}$ va decreciendo, lo que prueba el teorema previamente formulado.

\section{RESULTADOS EN SIMULACIÓN}

Esta sección muestra diferentes resultados en simulación obtenidos con la estrategia de control propuesta (IHMPC + FF) aplicada a un campo solar térmico de placas planas. Además, estos resultados se comparan con los obtenidos con la estrategia convencional IHMPC con el objetivo de demostrar las ventajas que se obtienen en el rechazo de perturbaciones. Se debe mencionar que los controladores se implementaron de acuerdo a los problemas de optimización establecidos en la Sec- 
ción 3 , en el cual IHMPC+FF es el problema formulado en Problema 2 y IHMPC es el diseñado en el Problema 1. Además, con el fin de evidenciar las principales diferencias entre la estrategia de control propuesta (IHMPC + FF) y la estrategia convencional (IHMPC), en la Figura 1 se muestra un diagrama esquemático descriptivo que resume la forma en la que se implementaron ambas estrategias. Observe que el modelo de proceso es el mismo que el modelo interno del controlador, en el que se mantiene la simulación de caso nominal.

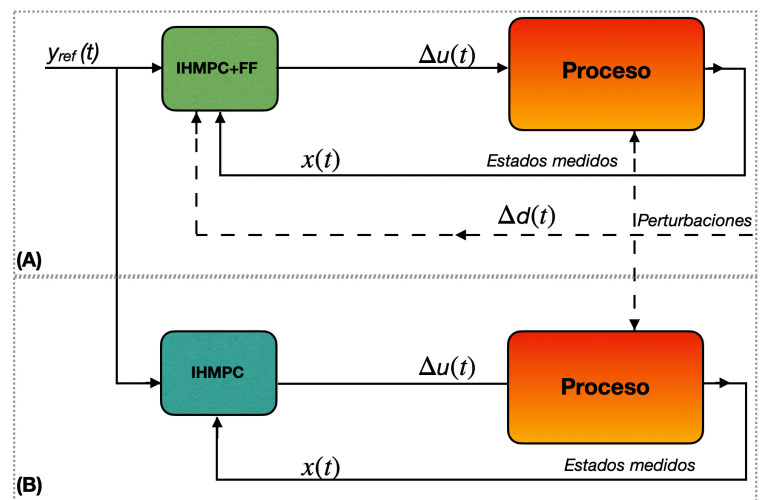

Figura 1: Estructuras de control IHMPC+FF (A) y $\operatorname{IHMPC~(B).~}$

Se debe comentar también que el campo solar térmico es un sistema de especial interés para la prueba de la técnica propuesta ya que sufre constantes perturbaciones debidos a los cambios naturales de la radiación y temperatura ambiente. De este modo, considerando el modelo linealizado del campo solar [2], la matriz de funciones de transferencia en el punto de operación elegido para las pruebas es:

$$
G(s)=\left[\begin{array}{llll}
\frac{-0,5304}{26,02 \cdot s+1} & \frac{0,02474}{26,02 \cdot s+1} & \frac{0,09728}{26,02 \cdot s+1} & \frac{0,9027}{26,02 \cdot s+1}
\end{array}\right]
$$

donde $y(s)=T_{\text {out }}(s)$ es la temperatura de salida del campo de captadores, y las entradas $\mathrm{y}$ perturbaciones son $u(s)=$ $\left[\Delta q(s), \quad \Delta I(s), \quad \Delta T_{a}(s), \quad \Delta T_{i n}(s)\right]^{\top}$, las cuales corresponden con el caudal, la radiación, la temperatura ambiente y la temperatura de entrada respectivamente. Los estados iniciales de la simulación se establecen como $u=$ $\left[6,96 \mathrm{~m}^{3} / \mathrm{h}, \quad 265,75 \mathrm{~W} / \mathrm{m}^{2}, \quad 13,07^{\circ} \mathrm{C}, \quad 41,87^{\circ} \mathrm{C}\right]^{\top}$ y $T_{\text {out }}=45,73^{\circ} \mathrm{C}$. Además, para la comparación de los controladores, ambos se sintonizaron con los mismos parámetros de ajuste los cuales son: $m=8, \mathbf{Q}_{y}=20 \operatorname{diag}(\mathrm{m}), \mathbf{R}=10^{-3} \operatorname{diag}(\mathrm{m})$, $\mathbf{S}_{y}=10^{6}, \mathbf{u}_{\max }=13,06\left[\mathrm{~m}^{3} / \mathrm{h}\right], \mathbf{u}_{\min }=-3,94$ $\left[\mathrm{m}^{3} / \mathrm{h}\right]$ y $\boldsymbol{\Delta} \mathbf{u}_{\max }=-\boldsymbol{\Delta} \mathbf{u}_{\min }=1\left[\mathrm{~m}^{3} / \mathrm{h}\right]$.

La simulación se realizó con un tiempo de muestreo de $T_{s}=1 \mathrm{~s}$. Las variaciones en las pertur- baciones se implementaron con un orden de magnitud mayor que las que ocurren en experiencias reales con el objetivo de demostrar el buen desempeño de la estrategia propuesta. Por otro lado, solo se introdujeron variaciones puntuales en las perturbaciones con el objetivo de visualizar de una forma más clara su efecto. Así, en el instante $t=10 \mathrm{~s}$, se introdujo un pulso de perturbación por $30 \mathrm{~s}$, en el que la $I$ se redujo en $350 \mathrm{~W} / \mathrm{m}^{2}$, la $T_{a}$ se redujo en $2{ }^{\circ} \mathrm{C}$ y la $T_{i n}$ se redujo en $3{ }^{\circ} \mathrm{C}$. Finalmente, en el instante $t=70 \mathrm{~s}$ se introdujo un cambio en la referencia para probar el desempeño del controlador ante esta situación. De esta forma, la referencia de $T_{\text {out }}(t)$ se redujo en $1.5{ }^{\circ} \mathrm{C}$. Todo lo descrito se presenta en la Figura 2.

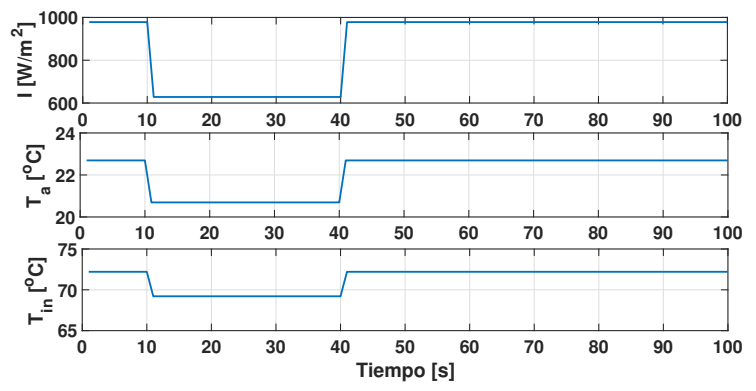

Figura 2: Variaciones en las perturbaciones durante la simulación.

La Figura 3 muestra las salidas del sistema y las entradas proporcionadas por cada controlador para el escenario de simulación descrito previamente. Como se puede apreciar, el controlador IHMPC+FF actuó un instante antes que el IHMPC en términos de $q(t)$ y $T_{\text {out }}(t)$. Esto demuestra que la compensación por adelanto de la técnica IHMPC+FF funcionó correctamente, rechazando las perturbaciones antes de que estas alteraran al sistema. Para el caso de seguimiento de referencias, ambos controladores funcionaron de forma idéntica ya que no hubo perturbaciones durante el cambio de referencias.

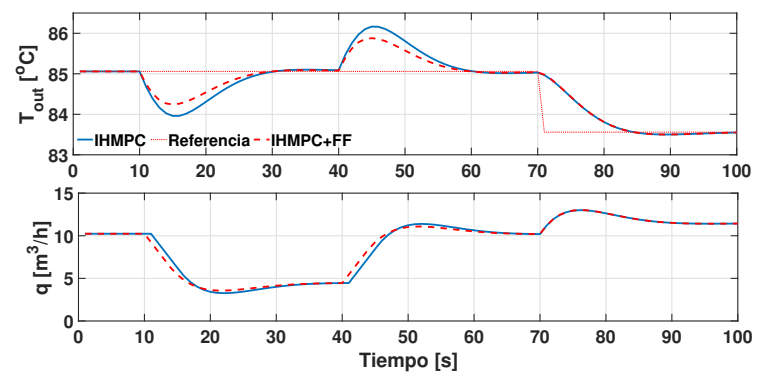

Figura 3: Entradas y salidas del sistema con los controladores IHMPC+FF y IHMPC.

Por otra parte, las Figuras 4 y 5 muestran la evolución de las variables de holgura y la función 
de coste para ambos controladores durante la simulación. Se debe remarcar que el parámetro de ajuste $S_{y}$ garantizó que las variables de holgura se usaran solo cuando fuera estrictamente necesario, siendo cero en estado estacionario (ver Figura 4). Además, se puede observar como la función de coste decreció asintóticamente (ver Figura 5).

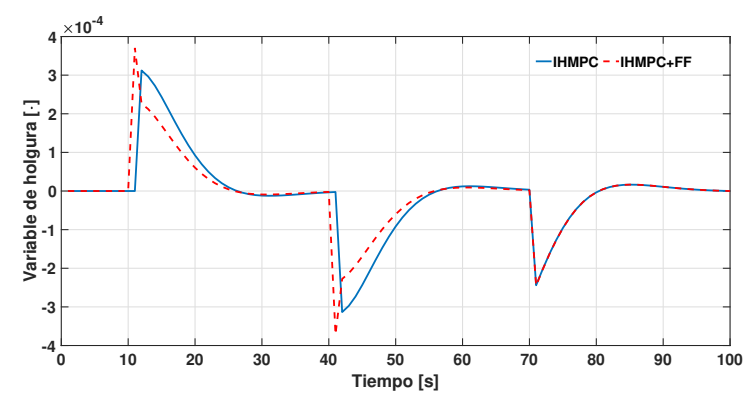

Figura 4: Evolución de las variables de holgura para el caso de IHMPC+FF y IHMPC durante la simulación.

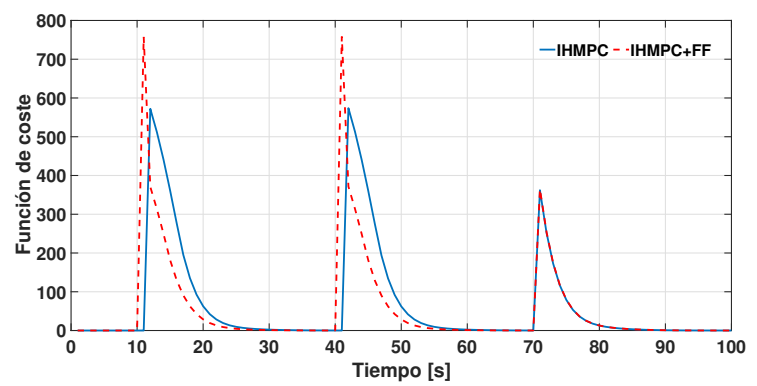

Figura 5: Evolución de la función de coste para el caso de IHMPC+FF y IHMPC durante la simulación.

Para cuantificar de forma numérica el desempeño de ambos controladores, en la Tabla 1 se presenta el valor de los índices IAE e ITAE para la simulación mostrada. El primer índice se calculó considerando la magnitud del error durante toda la simulación, mientras que en el segundo se consideró solo el tiempo durante el cual se produjo el error. Como se puede apreciar, el controlador IHMPC+FF presentó mejores resultados para ambos índices, con un valor de IAE del $10.74 \%$ y de ITAE del $15.07 \%$ más bajo que el IHMPC.

Cuadro 1: Índices de rendimiento para los controladores IHMPC+FF y IHMPC.

\begin{tabular}{l|ll} 
& IHMPC+FF & IHMPC \\
\hline ITAE & $1.24 \cdot 10^{3}$ & $1.46 \cdot 10^{3}$ \\
IAE & 29.10 & 32.60
\end{tabular}

\section{CONCLUSIONES}

Este trabajo presenta un controlador predictivo con compensación por adelanto y garantía de estabilidad para sistemas estables. En concreto, se desarrolla una estrategia MPC de horizonte infinito que garantiza la estabilidad en bucle cerrado y que compensa las perturbaciones que afectan al sistema en una única capa de control. La estabilidad del controlador se garantiza utilizando los conceptos de solución recursiva y decrecimiento asintótico de la función de coste. La técnica propuesta se prueba en simulación usando como planta de referencia un campo solar de captadores de placa plana. Además, se presenta una comparación con la estrategia convencional MPC de horizonte infinito pero sin incluir la compensación por adelanto. Los resultados obtenidos evidencian que la estrategia propuesta mejora el desempeño del controlador rechazando adecuadamente las perturbaciones al mismo tiempo que mantiene las características nominales de estabilidad, lo que asegura la convergencia en bucle cerrado tanto para los problemas de seguimiento de referencia como de rechazo de perturbaciones.

\section{AGRADECIMIENTOS}

Los autores agradecen el soporte económico del Consejo Nacional de Desarrollo Científico y Tecnológico (CNPq, Brasil) que ha financiado parte de este trabajo bajo la beca con código 201143/2019 - 4. Además, este trabajo ha sido desarrollado en el marco del proyecto "Microrredes para el autoabastecimiento solar de entornos productivos aislados (Microprod-Solar)" con código PCI2019-103378, financiado por la Agencia estatal de Investigación del Gobierno de España y por el Programa Iberoamericano de Ciencia y tecnología para el desarrollo (CYTED). 
English summary

\section{A stabilizing predictive controller with feedforward action: Preliminary results}

\begin{abstract}
This paper presents a nominal stabilizing predictive controller with a feedforward action for stable systems. An infinite horizon model predictive controller is developed to guarantee closed-loop stability and compensating disturbances in a single layer controller. The controller stability is assured using the recursive solution and the asymptotical decreasing of the cost function concepts. The infinite horizon predictive controller with feedforward action is implemented in a simulation scenario of a solar collector field in which it is compared to the same control approach without the disturbance compensations. It is demonstrated that the novel strategy can improve the control performance by correctly rejecting the disturbances and keeping the nominal stability features, leading to the convergence of the closed-loop system both for the reference tracking and measured disturbances scenarios.
\end{abstract}

Keywords: Model predictive controller, feedforward action, infinite horizon predictive controller, solar collector field.

\section{Referencias}

[1] A. Alsharkawi and J. A. Rossiter. Dual mode MPC for a concentrated solar thermal power plant. IFAC-PapersOnLine, 49(7):260$265,2016$.

[2] E. F. Camacho, M. Berenguel, and F. R. Rubio. Advanced Control of Solar Plants. Springer, London, England, 2012.

[3] J. D. Gil, L. Roca, and M. Berenguel. Modelling and automatic control in solar membrane distillation: Fundamentals and proposals for its technological development. Revista Iberoamericana de Automática e Informática industrial, 17(4):329-343, 2020.
[4] A. H. González, E. J. Adam, M. G. Marcovecchio, and D. Odloak. Application of an extended IHMPC to an unstable reactor system: Study of feasibility and performance. Journal of Process Control, 21(10):1493-1503, 2011.

[5] G. Marchante, A. Acosta, A. González, J. Zamarreño, and V. Álvarez. Evaluación de restricciones de confort en controlador predictivo para la eficiencia energética. Revista Iberoamericana de Automática e Informática industrial, 18(2):146-159, 2021.

[6] D. Q. Mayne. Model predictive control: Recent developments and future promise. Automatica, 50(12):2967-2986, dec 2014.

[7] D. Q. Mayne, J. B. Rawlings, C. V. Rao, and P. O. Scokaert. Constrained model predictive control: Stability and optimality. Automatica, 36(6):789-814, 2000.

[8] M. Pasamontes, J. Álvarez, J. Guzmán, and M. Berenguel. Hybrid modeling of a solar cooling system. IFAC Proceedings Volumes, 42(17):26 - 31, 2009. 3rd IFAC Conference on Analysis and Design of Hybrid Systems.

[9] J. B. Rawlings and K. R. Muske. The stability of constrained receding horizon control. IEEE Transactions on Automatic Control, 38:1512 - 1516, 1993.

[10] M. A. Rodrigues and D. Odloak. MPC for stable linear systems with model uncertainty. Automatica, 39(4):569-583, 2003.

[11] J. A. Rossiter. Model-based predictive control: a practical approach. Press, CRC, 1 edition, 2003.

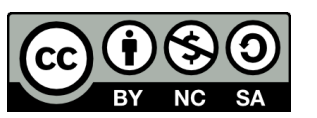
(C) 2021 by the authors. Submitted for possible open access publication under the terms and conditions of the Creative Commons Attribution CC BY-NC-SA 4.0 license (https://creativecommons.org/licenses/by-ncsa/4.0/deed.es). 